\title{
A NOTE ON YOUNG INEQUALITY
}

\author{
J. JAKŠETIĆ AND J. PEČARIĆ
}

Abstract. In this paper we give an extension of Young inequality establishing lower and upper bound.

Mathematics subject classification (2010): 26D15.

Keywords and phrases: Hölder inequality, Monotone function, Young inequality.

\section{REFERENCES}

[1] Olympiad Math. Madness, Analysis, Available online at http://www.geocities.com/CapeCanaveral/Lab/4661/

[2] J. E. PeČarić, F. Proschan And Y. C. Tong, Convex functions, Partial Orderings and Statistical Applications, Academic Press, New York, 1992.

[3] F. QI, A. Hoorfar, A new refinement of Young's inequality, Math. Inequal. Appl. 11, 4 (2008), 689692.

[4] W. H. Young, On classes of summable functions and their Fourier series, Proc. Roy. Soc. London, Ser. A 87 (1912), 225-229. 